\title{
Anomalous phase dynamics of driven graphene Josephson junctions
}

\author{
S. S. Kalantre $\odot,{ }^{1}$ F. Yu $\odot,{ }^{1}$ M. T. Wei $\odot,{ }^{1}$ K. Watanabe $\odot,{ }^{2}$ T. Taniguchi, ${ }^{2}$ M. Hernandez-Rivera $\odot,{ }^{3}$ \\ F. Amet, ${ }^{3}$ and J. R. Williams ${ }^{1, *}$ \\ ${ }^{1}$ Joint Quantum Institute and the Maryland Quantum Materials Center, Department of Physics, University of Maryland, \\ College Park, Maryland 20742, USA \\ ${ }^{2}$ National Institute for Materials Science, 1-1 Namiki, Tsukuba 305-0044, Japan \\ ${ }^{3}$ Department of Physics and Astronomy, Appalachian State University, Boone, North Carolina 28608, USA
}

(Received 5 November 2019; revised manuscript received 9 March 2020; accepted 18 March 2020; published 28 April 2020)

\begin{abstract}
Josephson junctions with weak links of exotic materials allow the elucidation of the Josephson effect in previously unexplored regimes. Further, such devices offer a direct probe of novel material properties, for example, in the search for Majorana fermions. In this paper, we report on dc and ac Josephson effect of high-mobility, hexagonal boron nitride encapsulated graphene Josephson junctions. On the application of $\mathrm{rf}$ radiation, we measure phase-locked Shapiro steps. An unexpected bistability between \pm 1 steps is observed with switching times on the order of seconds. A critical scaling of the bistable state is measured directly from the switching time, allowing for direct comparison to numerical simulations. We show such intermittent chaotic behavior is a consequence of the nonlinear dynamics of the junction and has a sensitive dependence on the current-phase relation. This paper draws connections between nonlinear phenomena in dynamical systems and their implications for ongoing condensed matter experiments exploring topology and exotic physics.
\end{abstract}

DOI: 10.1103/PhysRevResearch.2.023093

\section{INTRODUCTION}

The ground-state wave function of a superconductor is endowed with an emergent phase on the macroscopic scale [1]. Josephson pointed out that two superconductors separated by a nonsuperconducting weak link produce a supercurrent that depends on the phase difference between the superconductors [2]. Today, eponymously known as the Josephson effect, it remains a hallmark of the superconducting state and has led to a large number of scientific and technological advances [3-5]. As one of the few phase-sensitive devices available in condensed matter physics, it has found use in the investigation of novel superconducting materials [6] and more recently in the identification of topological superconductors and Majorana bound states [7-12]. In particular, the Josephson effect in the presence of $\mathrm{rf}$ radiation (the ac Josephson effect) has been instrumental in advancing our knowledge of underlying physics in these systems. The incorporation of high-quality, tunable two-dimensional materials into a Josephson junction (JJ) now allows for these nonlinear devices to be probed in previously unexplored regimes. As the JJ becomes more prevalent in use both as a detector of novel material properties and exotic circuit elements, a thorough understanding of the role of nonlinear dynamics in these devices is essential.

\footnotetext{
*Corresponding author: jwilliams@physics.umd.edu

Published by the American Physical Society under the terms of the Creative Commons Attribution 4.0 International license. Further distribution of this work must maintain attribution to the author(s) and the published article's title, journal citation, and DOI.
}

The understanding of JJs driven by externally applied currents and radio-frequency (rf) radiation rests on simple circuit models that capture the superconducting phase dynamics. The relationship between the supercurrent $(I)$ and the phase difference across the $\mathrm{JJ}(\phi)$ is encoded in a current-phase relationship (CPR), which subsumes the microscopic physics of the weak link [13]. The dynamics of a JJ then follow the evolution of a phase particle in an effective potential dependent on the physical parameters of the junction, such as normal-state resistance, capacitance, and externally applied currents $[14,15]$. At the heart of such models is a dynamical system described by a set of ordinary differential equations made nonlinear by the CPR $[1,16]$.

Nonlinear dynamical systems are interesting in their own right because of the rich set of phenomena they display. The phase dynamics of a driven $\mathrm{JJ}$ is similar to that of a driven, damped pendulum - a system known to be chaotic. Nonlinear effects in JJs have been studied extensively in theory [17-23]. As a result, a plethora of nonlinear effects including chaos, intermittency, and strange attractors can occur [24], and these effects should manifest in JJs. Nonlinear effects in JJs also have important implications for the use of the ac Josephson effect in JJs with topological material used as a weak link. Unlike conventional JJs, these topological JJs have $4 \pi$-periodic CPR. The ac Josephson effect has been proposed as a means to detect topological superconductivity, where the $4 \pi$-periodic CPR results in a doubling of the Shapiro step height [7-9]. While this is an effective and reliable signature for overdamped JJs, nonlinear effects in moderately and heavily damped junctions can produce a period doubling bifurcation [24], which would emulate the effects expected in a topological JJ. As the range and quality of materials 
employed to investigate topological superconductivity expand, a study exploring the parameter space of JJs under external rf excitation is timely. It is critical to detail nonlinear and chaotic phenomena in JJs to ensure these effects do not masquerade as any exotic effects that could erroneously be ascribed to the novelty of the weak link material.

In this paper, we draw a bridge between experiments on graphene JJs and the unexpected consequences of the nonlinear phase dynamics. Graphene encapsulated in hexagonal boron nitride (h-BN) offers a platform to fabricate junctions with ballistic carrier transport. Moreover, gate electrodes and magnetic fields can be used to tune the junction parameters. We focus on measurements of the junction resistance as a function of a dc current bias and rf power, referred to as Shapiro diagrams. Measurements of the Shapiro diagram show strong deviations from the expected behavior of overdamped JJs. These deviations can be used to identify nonlinear dynamics in the JJ. Focus is given, and portions of these diagrams disappear in certain regimes, a result of chaotic behavior possessing timescales many orders of magnitude slower than have ever been observed before in JJs. This slow timescale allows for this nonlinear effect to be tracked in real time. Through comparison to simulations, the timescale is shown to depend sensitively on the CPR of the junction, becoming large for skewed CPRs like that predicted for weak link materials possessing a Dirac (linear) energy dispersion. These results should inform future experiments using rf radiation to probe exotic materials in JJs [7-9] and devices that exploit JJs in rf environments [25,26].

\section{DEVICE CHARACTERISTICS}

$\mathrm{H}-\mathrm{BN}$ encapsulated graphene is fabricated into JJs, proximitized with molybdenum-rhenium alloy (MoRe) superconducting leads [Fig. 1(a)]. MoRe is a type-II superconductor with an upper critical field of $8 \mathrm{~T}$. Previously, such junctions have been used to study supercurrent in the quantum Hall regime and have been shown to have ballistic carrier transport [27]. The junction length along the direction of current flow is $\sim 500 \mathrm{~nm}$ and the width is $\sim 2.7 \mu \mathrm{m}$. In addition to MoRe contacts, we also have $\mathrm{Cr} / \mathrm{Au}$ top gates and a backgate to electrically tune electron density. The top gates are kept grounded for the entirety of this experiment.

The device was cooled down in a cryostat with a base temperature of $50 \mathrm{mK}$. The magnetic field $B$ was applied out of plane and perpendicular to the junction. Four terminals at the chip level measure the $\mathrm{JJ}$ in a two-terminal geometry (which removes the series resistance of the wires down the cryostat). The differential resistance $R=\frac{d V}{d I}$ is measured with a lock-in amplifier at a frequency of $19 \mathrm{~Hz}$ and excitation of $1 \mathrm{nA}$ as a function of back gate voltage $\left(V_{\mathrm{BG}}\right)$ and the applied dc current bias $\left(I_{\mathrm{dc}}\right)$. Figure $1(\mathrm{~b})$ shows the $V_{\mathrm{BG}}$ dependence of the critical current. The charge neutrality point (CNP) is found to be at $V_{\mathrm{BG}}=-0.75 \mathrm{~V}$, corresponding to the lowest critical current and the largest normal-state resistance. Figure 1(c) shows the Fraunhofer pattern $R(B)$ at the CNP.

\section{MEASUREMENT RESULTS}

In order to probe the phase dynamics of the $\mathrm{JJ}, R$ is measured as a function of $I_{\mathrm{dc}}$ and $\mathrm{rf}$ power. When junctions are (a)
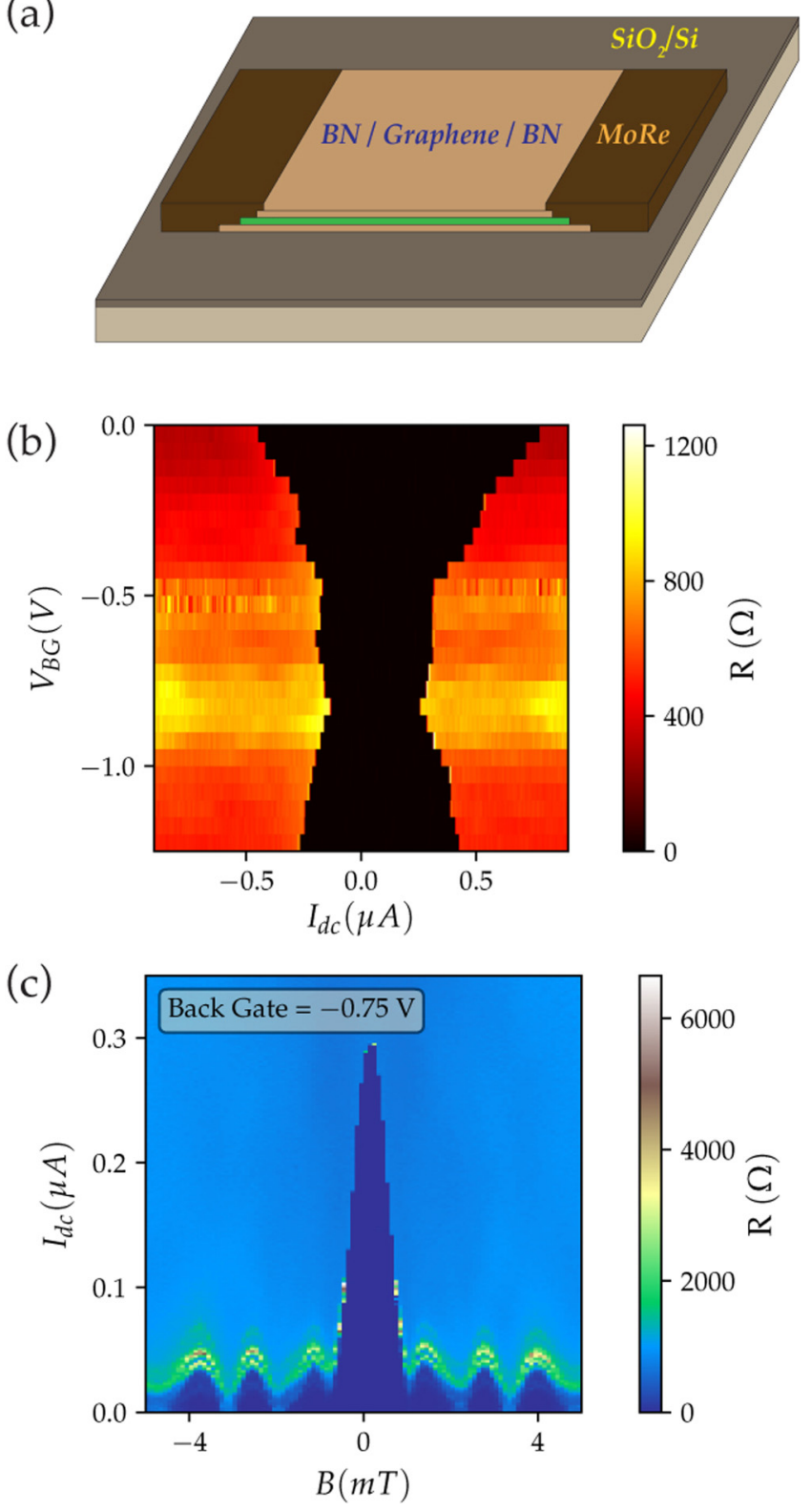

FIG. 1. (a) A schematic of the h-BN encapsulated graphene device. Side contacts to graphene are made with molybdenum-rhenium (MoRe) as the superconductor. The device is fabricated on $\mathrm{SiO}_{2} / \mathrm{Si}$ substrate and can be backgated. (b) Dependence of the critical current on $V_{\mathrm{BG}}$ near the CNP, which occurs at $V_{\mathrm{BG}}=-0.75 \mathrm{~V}$. (c) Magnetic diffraction (Fraunhofer) pattern for the junction at CNP. The periodicity implies a junction area of about $1.5 \mu \mathrm{m}^{2}$.

periodically driven, phase-locked solutions produce a fixed voltage across the JJ. These are known as Shapiro steps [28], and show up as zero differential resistance in a lock-in measurement. The measurement of the Shapiro diagram yields information about the CPR and has been used previously to study weak links of topological materials [7,8,10-12,29-31].

\section{A. Shapiro diagram and broken node}

Figure 2(a) shows a Shapiro diagram at rf frequency $(f)$ of $6.350 \mathrm{GHz}$ and $V_{\mathrm{BG}}=-0.75 \mathrm{~V}$. We observe regions of zero differential resistance corresponding to Shapiro steps 

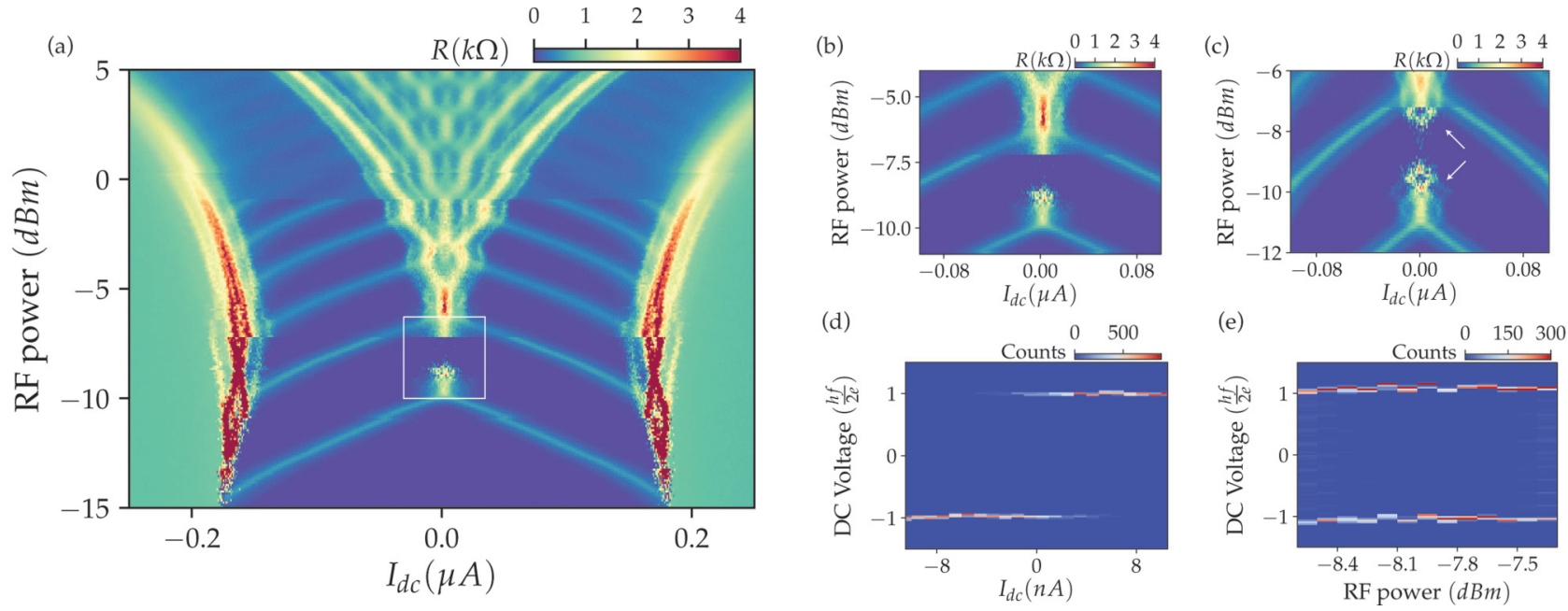

FIG. 2. (a) Shapiro diagram at $f=6.350 \mathrm{GHz}, V_{\mathrm{BG}}=-0.75 \mathrm{~V}$, and $B=0$. The first elongated note at the lowest rf power is also broken (white box). (b) A close-up of the broken node region of (a). (c) Shapiro diagram at $f=6.350 \mathrm{GHz}, V_{\mathrm{BG}}=-0.55 \mathrm{~V}$, and $B=0.3 \mathrm{mT}$. The broken node shifts in position and extra features are observed (white arrows). (d, e) Direct dc voltage, binned in regular time intervals, around the broken node as a function of $I_{\mathrm{dc}}$ and $\mathrm{rf}$ power. The measured voltage values cluster around two voltages and overlap, indicating a bistability between the \pm 1 Shapiro steps.

separated by resistive transitions between steps. This Shapiro diagram is distinct in two principal ways from previous measurements on graphene [32] and other materials [7,8,1012,29-31]. The first is the resistive transitions, which are elongated nodelike regions, not single points that are expected for the Bessel function step width as a function of rf power [4]. These elongated nodes are a generic feature of underdamped JJs (explained below) and can be used to infer underdamped behavior even when samples lack hysteresis in measurements of a $I-V$ curve. Second, the first node separating the \pm 1 steps at $I_{\mathrm{dc}}=0$ disappears (indicated by the white box) for certain rf powers. Switching behavior on a timescale slower than the integration time for a lock-in measurement can lead to such a feature. Since the integration time used was around $1 \mathrm{~s}$, this implies a switching timescale on the order of seconds. Figure 2(b) shows a closeup of the broken node region. In addition to the broken node, the details of resistive transition are dependent on the junction parameters. Figure 2(c) shows the changes when $I_{\mathrm{dc}}$ is reduced by $B$, where additional structure in $R$ is observed (white arrows). For a more extensive visualization of the changes to the broken node region in response to small changes in $f, V_{\mathrm{BG}}$, and $B$, see Appendix A.

To investigate the broken node region further, we switch to direct dc voltage measurements as a function of time. Figures 2(d) and 2(e) show a histogram of the voltage values measured as a function of $I_{\mathrm{dc}}$ and $\mathrm{rf}$ power. For a given $I_{\mathrm{dc}}$ or $\mathrm{rf}$ power, we measure the dc voltage $V(t)$ at regular time intervals. These values are then binned. The counts are reported as the color in Figs. 2(d) and 2(e). When the count distribution is bimodal, a bistability between the two values can be inferred. We clearly observe a bistability between the two \pm 1 Shapiro steps at the broken node as a function of $I_{\mathrm{dc}}$ [Fig. 2(d)] and rf power [Fig. 2(e)].

\section{B. Bistability and switching time}

Figure 3(a) shows the evolution of $V(t)$ with $I_{\mathrm{dc}}$ from negative to positive values. For large negative values $(\approx-10 \mathrm{nA})$ of $I_{\mathrm{dc}}$, the junction is phase locked to -1 ; the same occurs for large positive values of $I_{\mathrm{dc}}$. However, in the transition region between -1 and +1 , we see a bistable region. $V(t)$ randomly switches between \pm 1 steps. $\tau$ is defined as the average time $V(t)$ spends on a particular step. $\tau$ is extraordinarily long — on the order of seconds-much longer than any junction timescales. For comparison, a typical timescale associated with $\mathrm{JJ}$ dynamics is the characteristic frequency of the junction, defined as $f_{c}=\frac{2 e I_{c} R}{\hbar}$, where $I_{c}$ is the critical current and $R$ is the normal-state resistance. $f_{c}$ for this junction is $\approx 50 \mathrm{GHz}$. Figure $3(\mathrm{~b})$ shows the dependence of $\tau$ on $I_{\mathrm{dc}} . \tau$ changes by almost three orders of magnitude in the bistable (a)

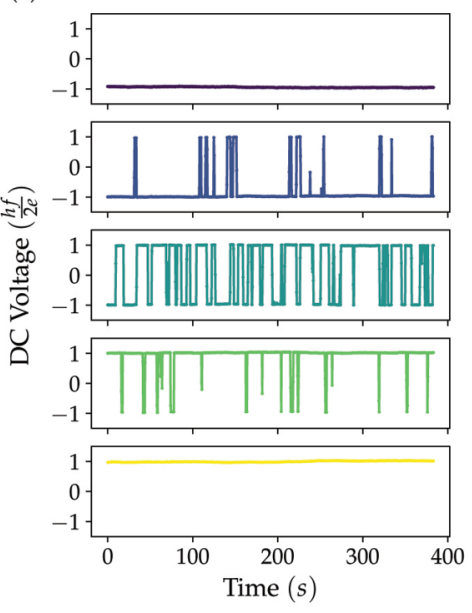

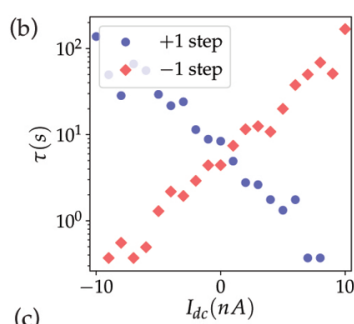

(c)

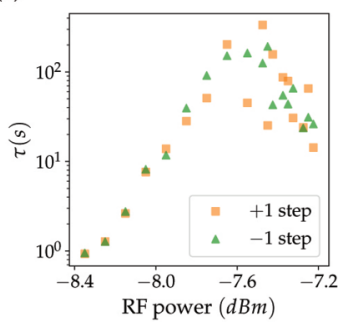

FIG. 3. (a) Dc voltage measured as a function of time, measured at $I_{\mathrm{dc}}$ values of $\{-14,-3,1,5,14\} \mathrm{nA}$ (top to bottom). A change from being entirely on the -1 to the +1 Shapiro step is seen. Near zero current bias, the voltage stochastically switches between the two steps. A long switching timescale, of order $10 \mathrm{~s}$, is observed. (b, c) Dependence of the switching timescale $\tau$ on $I_{\mathrm{dc}}$ and rf power measured in the region of the broken node. 
(a)
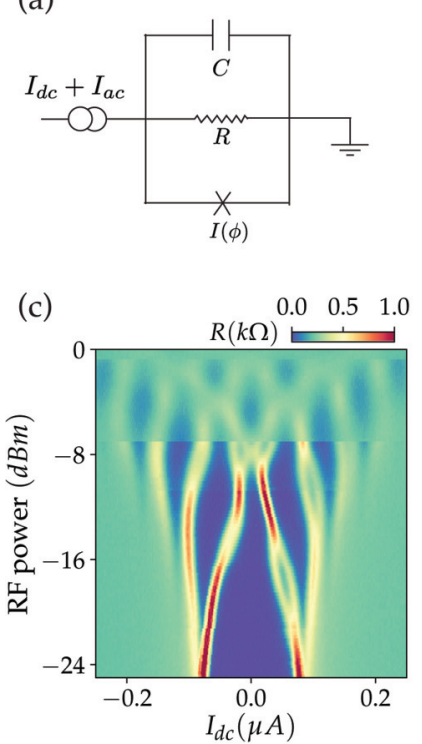
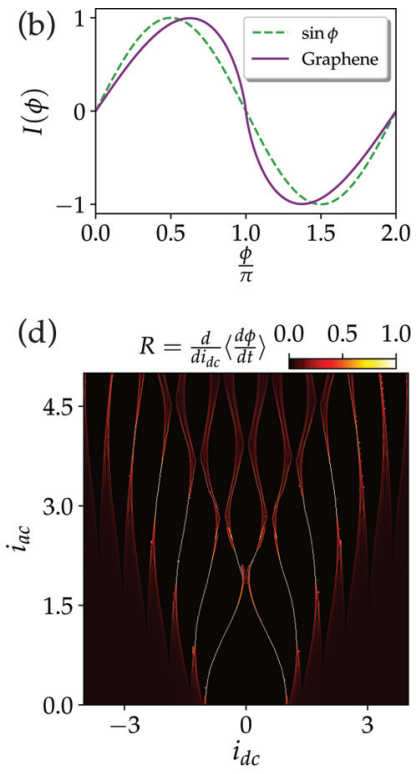

FIG. 4. (a) Circuit diagram of the RCSJ model. A supercurrent the phase dependence of which is given by CPR is assumed to flow in parallel to a resistor and a capacitor. External dc and ac currents can be used to drive the junction. (b) A comparison between graphene and a sinusoidal CPR. (c) Shapiro diagram measured at $6.350 \mathrm{GHz}$, $V_{\mathrm{BG}}=+0.25 \mathrm{~V}$, and magnetic field set to $0.7 \mathrm{mT}$. (d) Simulated Shapiro diagram for $Q=1$ and $\Omega=0.6$ which qualitatively matches the features seen in (c).

region. A similar scaling of $\tau$ now as a function of $\mathrm{rf}$ power is shown in Fig. 3(c). Note the $\log$ scale on the $\tau$ axis in Figs. 3(b) and 3(c).

Such scaling behavior is unexpected. One would expect that the dynamics smoothly change between \pm 1 steps. The scaling with rf power also shows a maximum in $\tau$ at around $-7.4 \mathrm{~dB} \mathrm{~m}$ in Fig. 3(c) and quickly decays away from this maximum. These observations point out that it is crucial to understand the exact nature of the phase-locked solutions though a model, which we do in the following section.

\section{RESISTIVELY AND CAPACITIVELY SHUNTED JUNCTION MODEL}

A model for the evolution of $\phi(t)$ under external $\mathrm{dc}$ and rf drives can be used to understand the Shapiro diagram. A simple circuit model for JJs, called the resistively and capacitively shunted junction (RCSJ) model [Fig. 4(a)] [14,15], has been widely used for understanding phase dynamics in JJs. In this section, we describe the parameters for our model and the modifications required when graphene serves as the weak link. $\phi$ can be related to experiment by calculating voltage across the junction using the Josephson relation $V=\frac{\hbar}{2 e} \frac{d \phi}{d t}[2]$.

\section{A. Model definition}

In junctions with a conducting weak link, the supercurrent flow is mediated by Andreev bound states (ABSs), formed by Andreev reflection at the two weak link-superconductor interfaces $[33,34]$. The position of ABSs depends on $\phi$, the electron/hole dispersion in the weak link region, and the

coupling strength between the superconductors and the weak link. For a short and wide ballistic graphene junction at the Dirac point, the CPR is [16]

$$
\begin{aligned}
I(\phi) & =\frac{e \Delta}{\hbar} \frac{2 W}{\pi L}\left(\cos \frac{\phi}{2}\right) \tanh ^{-1}\left(\sin \frac{\phi}{2}\right) \\
& =I_{c} \frac{2}{1.33}\left(\cos \frac{\phi}{2}\right) \tanh ^{-1}\left(\sin \frac{\phi}{2}\right)
\end{aligned}
$$

where $\Delta$ is the superconducting order parameter, $W$ is junction width perpendicular to current flow, and $L$ is the junction length. The numerical factor of $\frac{2}{1.33}$ ensures that the maximum as a function of $\phi$ is $I_{c}$. See Fig. 4(b) for a plot of this CPR. Although there is not sufficient evidence that the $\mathrm{JJ}$ is in the ballistic short junction regime, we use the form given by Eq. (1) in the following analysis given that it is analytic and allows us to comment on the relevant physics in this system.

Once the CPR is established, the phase dynamics can be modeled by the RCSJ model and the governing equation is

$$
\frac{\hbar C}{2 e} \frac{d^{2} \phi}{d t^{2}}+\frac{\hbar}{2 e R} \frac{d \phi}{d t}+I(\phi)=I_{\mathrm{dc}}+I_{\mathrm{ac}} \sin (\omega t)
$$

where $C$ is the junction capacitance, $R$ is the normal-state resistance, and $I_{\mathrm{dc}}$ and $I_{\mathrm{ac}}$ are the externally applied dc and ac currents. The equation can be cast into dimensionless form by transforming $t \rightarrow t \sqrt{\frac{2 e I_{c}}{\hbar C}}$ :

$$
\frac{d^{2} \phi}{d t^{2}}+\frac{1}{Q} \frac{d \phi}{d t}+i(\phi)=i_{\mathrm{dc}}+i_{\mathrm{ac}} \sin (\Omega t)
$$

where $Q=\sqrt{\frac{2 e I_{c} R^{2} C}{\hbar}}$ is called the quality factor and $\Omega=$ $\omega \sqrt{\frac{\hbar C}{2 e I_{c}}}$ is the normalized frequency. Depending on the value of the capacitance and consequently the $Q$ value, the junction can be overdamped $(C \rightarrow 0 \therefore Q \rightarrow 0)$ or underdamped $(Q \gtrsim 1)$.

An equivalent first-order autonomous form $\frac{d y}{d t}=F(y)$ can be obtained by defining $y=\left(\phi, \frac{d \phi}{d t}, \Omega t\right)$. This implies that the phase space for this system has to be three dimensional, and hence solutions are not limited to fixed points and limit cycles, but strange attractors and chaotic effects are possible (Poincaré-Bendixson theorem [24,35]). On the other hand, in the limit $C \rightarrow 0$, the second-order term vanishes and we are left with two-dimensional phase space, where the dynamics is restricted to fixed points and limit cycles. In the $C \rightarrow 0$ limit, we recover the overdamped or resistively shunted junction (RSJ) model, the dynamics of which are well understood. See Appendix B for a discussion on Shapiro diagrams in the overdamped limit.

The flow on $y$ defined by $F(y)$ has a Jacobian equal to $\frac{-1}{Q}$ implying a dissipative system. It can be shown that in the presence of dissipation the dynamics of a JJ with a sinusoidal CPR is equivalent to the driven pendulum. This correspondence to nonlinear dynamics of the driven-damped pendulum has been explored before in simulations and connections to $\mathrm{JJ}$ experiments have been drawn, as we elaborate on in the next subsection. 


\section{B. Previous work on underdamped, driven chaotic systems}

Early motivation to consider the nonlinear dynamics of Josephson junctions arose from the large values of electric noise observed in experiment. It was observed that JJ-based parametric amplifiers resulted in broadband voltage fluctuations [36] with a noise temperature on the order of $10^{4} \mathrm{~K}$, which was ascribed to the chaotic behavior of Eq. (4) [17]. It was suggested that this arises from the presence of a strange attractor in phase space for a certain set of parameters characterizing the junctions.

Experimentally, Poincaré maps and bifurcations present in the parameter space were first measured with phase-locked electrical loops the dynamics of which are similar to JJs and the driven simple pendulum equation [21]. A similar system was used to investigate symmetry breaking in solutions of the equation as a period-doubling route to chaos. Chaotic and intermittency was reported in measurements of bifurcation cascades of phase-locked loops [37]. Intrinsic and noiseinduced intermittency due to crisis has been been studied with numerical simulations. The authors reported an intermittent switching behavior between positive and negative voltage solutions with a characteristic power-law-type relation for the switching timescale [38]. The basin boundary for these solutions was found to be fractal.

The spontaneous presence of finite dc voltage steps at zero bias under rf radiation has been observed before and was dubbed the inverse ac Josephson effect [39]. The switching between two voltage steps was predicted to occur in the limit of high junction capacitance and was observed. A switching timescale on the order of minutes was measured: this feature was proposed as a voltage standard.

Extensive numerical simulations were carried to investigate the chaotic behavior of JJs and the effect of noise $[18,19]$. In experiments with a Josephson junction, a correlation was found between the noise and the fractal dimension of the boundary between periodic solutions [40,41]. Despite the extensive numerically simulations of the driven pendulum system, experimental verification remains scarce and is restricted to qualitative comparison [41-44].

\section{Simulation results for the underdamped RCSJ model}

We now describe the simulation of a Shapiro diagram under the RCSJ model. The voltage across the junction is given by $V(t)=\frac{\hbar}{2 e} \frac{d \phi}{d t}$. Since the voltage is measured in the dc limit, the average voltage is given as

$$
\begin{aligned}
\frac{2 e}{\hbar}\langle V\rangle_{n} & =\left.\left\langle\frac{d \phi}{d t}\right\rangle\right|_{n} \\
& =\frac{1}{n T} \int_{0}^{n T} \frac{d \phi}{d t} d t \\
& =\frac{\phi(n T)-\phi(0)}{n T}
\end{aligned}
$$

where $T=\frac{2 \pi}{\Omega}$ is the drive period and $n$ is a large number to average out transient behavior. For a phase-locked solution, $\phi$ advances by an integer number of $2 \pi$ period, and $\left\langle\frac{d \phi}{d t}\right\rangle$ at the $m t h$ Shapiro step is given by $m \Omega$. The dc limit is achieved when $n \rightarrow \infty$. It is also useful to define a window average for a a fixed number of periods which we denote as $\langle V\rangle_{n}=\left.\left\langle\frac{d \phi}{d t}\right\rangle\right|_{n}$. We will use both the dc limit and fixed window averages in the following sections. The values of $\langle V\rangle_{n}=\left.\left\langle\frac{d \phi}{d t}\right\rangle\right|_{n}$ are normalized in units of $\Omega$ so that the $m t h$ Shapiro step corresponds to $\langle V\rangle=\left.\left\langle\frac{d \phi}{d t}\right\rangle\right|_{n}=m$. It is also important to consider the initial conditions for solving the ordinary differential equation given by Eq. (4). Since it is of second order, a unique solution requires two initial numbers and we use the initial condition $(\phi(t=0), \dot{\phi}(t=0))=(0,0)$ unless otherwise stated. In order to understand the effects of noise, a Wiener process in time is added to the right-hand side of Eq. (4). This models a Gaussian white current noise source with zero mean. As a result, the RCSJ model with noise becomes a stochastic differential equation (SDE) as follows:

$$
\frac{d^{2} \phi}{d t^{2}}+\frac{1}{Q} \frac{d \phi}{d t}+i(\phi)=i_{\mathrm{dc}}+i_{\mathrm{ac}} \sin (\Omega t)+\sigma_{n} \eta(t)
$$

where $\eta(t) d t$ is a Weiner process and $\sigma_{n}$ controls the noise strength. It is useful to think of $\sigma_{n}^{2}$ as being proportional to an energy or temperature scale, which would hold exactly if the noise sources were due to Johnson-Nyquist noise in resistors. In simulations with noise, we integrate this SDE with the Euler-Maruyama method.

To understand our experimental results, we consider junctions in the weakly underdamped limit where $Q \approx 1$. Since the junction capacitance is not measured directly, we indirectly infer that the value of $Q$ must be close to 1 . Figure 4(c) shows a Shapiro diagram measured at $6.350 \mathrm{GHz}, V_{\mathrm{BG}}=$ $+0.25 \mathrm{~V}$, and magnetic field of $0.7 \mathrm{mT}$. We see that results are different from those at CNP and the broken node is not present. Figure 4(d) shows a simulated Shapiro diagram with $Q=1$ and $\Omega=0.6$; qualitative reproduction of the measured features is observed. In particular, the contours separating the steps meet in points ( \pm 1 steps) or show a small gap between them. Since the values for $V_{\mathrm{BG}}$ and $B$ chosen in Fig. 4(c) cause a reduction in $R$ and $I_{c}$ and hence a reduction in $Q$, we expect the dynamics at CNP and zero magnetic field to be described by a model with $Q>1$.

Figure 5(a) shows a simulated Shapiro diagram for $Q=$ 1.5 and $\Omega=0.5$. As opposed to diagrams in the overdamped limit (Appendix B), there are many distinctive features. The resistive contours for the steps meet in elongated notes as opposed to single points, similar to the experimentally observed features [Fig. 2(a)]. For a more detailed comparison between the fine features of the measurements and simulations, see Appendix C.

\section{Discrete symmetry of the underdamped RCSJ model}

We now focus on the explanation of the broken node feature. Since the node occurs on the $I_{\mathrm{dc}}=0$ line, we consider the following equation:

$$
\frac{d^{2} \phi}{d t^{2}}+\frac{1}{Q} \frac{d \phi}{d t}+i(\phi)=i_{\mathrm{ac}} \sin (\Omega t) .
$$

Equation (9) has a discrete symmetry among the possible solutions. For $\phi \rightarrow-\phi$ and $t \rightarrow t+\frac{\pi}{\Omega}$, the equation is left invariant. Note that we also need $i(\phi)=-i(-\phi)$ and this holds 

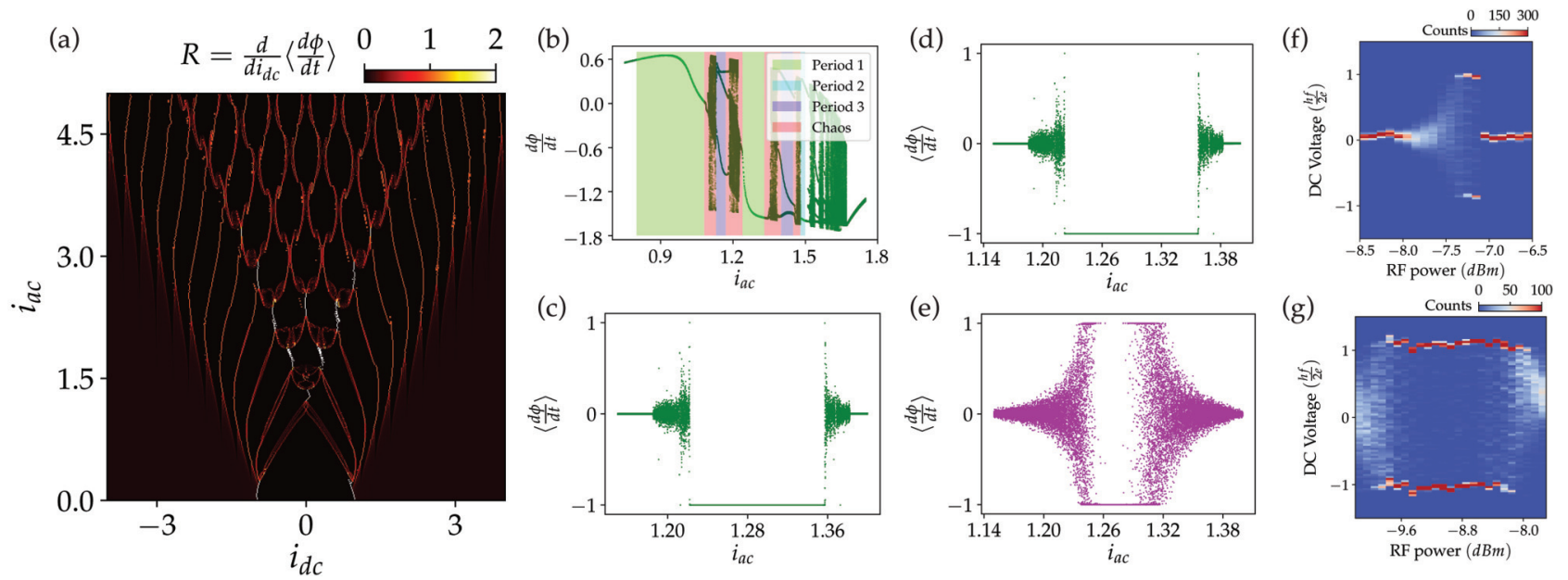

FIG. 5. (a) Shapiro diagram simulated for $Q=1.5$ and $\Omega=0.5$, and initial condition $(\phi(t=0), \dot{\phi}(t=0))=(0,0)$. Contours separating two steps have an intricate structure as opposed to point touchings. In particular, along the $i_{\mathrm{dc}}=0$ line, the contour separating \pm 1 steps is elongated. (b) Poincaré map $\phi(n T)$ for the same parameters as (a) with $i_{\mathrm{dc}}=0$ and $i_{\mathrm{ac}}$ varied in a range around the elongated node separating \pm 1 steps. Periodic attractors with different periods (corresponding to the number of points in the Poincaré map for a given $i_{\text {ac }}$ ), as well as chaotic windows separating them, are observed. (c) Dc limit of the Poincaré map with a window size of 200 periods. (d) A fine scan of the period 1 attractor of the +1 step. (e) Effect of noise on the attractor from (d). Both \pm 1 steps are possible solutions and a bistable region emerges. $(\mathrm{f}, \mathrm{g})$ Experimentally measured histograms of $V(t)$ values as a function of $\mathrm{rf}$ power at the broken node for $(f, B)=(6.322 \mathrm{GHz}, 0 \mathrm{mT})(\mathrm{f})$ and $(6.350 \mathrm{GHz}, 0.5 \mathrm{mT})(\mathrm{g})$.

from time-reversal symmetry [13]. This symmetry implies

$$
\phi(t) \text { is a solution } \Rightarrow-\phi\left(t+\frac{\pi}{\Omega}\right) \text { is a solution. }
$$

Under this operation, for given values of $Q, \Omega$, and $i_{\mathrm{ac}}$, if $\langle V\rangle=m$ is possible, so is $\langle V\rangle=-m$. Hence if $m \neq 0$, it is possible for the system to have two distinct solutions with an inequivalent observable (the dc voltage) in a measurement. Which of these two phase-locked solutions is achieved depends on the initial conditions $(\phi(t=0), \dot{\phi}(t=0))$. It is convenient to think of a basin structure [24] for each of the two solutions, where each initial condition eventually settles into one of these two possible solutions. We will later see how an interplay between the basin structure and the noise level can lead to the switching characteristics measured in Figs. 3(b) and $3(\mathrm{c})$.

\section{E. Poincaré maps and bistability}

A handy construction for elucidating the behavior of a dynamical system is a Poincaré map or section. For a dynamical system, it is the intersection of a periodic orbit with a lowerdimensional subspace, transverse to the flow of the system. In our case, for the RCSJ model, we consider $\left(\phi(t), \frac{d \phi}{d t}(t)\right)$ for $t=n \frac{2 \pi}{\Omega}$ where $n$ is an integer.

In Fig. 5(b), the Poincaré map for $\frac{d \phi}{d t}$ with parameters $Q=$ 1.5 and $\Omega=0.5$ is plotted. $i_{\mathrm{dc}}$ fixed to zero and $i_{\mathrm{ac}}$ is varied in a range around which the \pm 1 steps meet. The Poincaré map has a rich structure with periodic attractors, separated by windows where the dynamics causes $\frac{d \phi}{d t}$ to spread uniformly over a range of values. The behavior in these windows is similar to the periodic doubling bifurcation cascade in the logistic map [24] and we refer to these windows as chaotic windows. In general, the phase-space structure of the model along the $i_{\mathrm{ac}}$ axis consists of periodic attractors separated by chaotic windows.

The phase dynamics $\phi(t)$ is challenging to measure directly in an experiment and the only quantity accessible is the dc voltage. To this end, we now discuss the Poincaré map with an average over a fixed window. We simulate dynamics for 10000 periods, averaged over 200 consecutive periods, and plot the values taken by the window averaged $\left.\left\langle\frac{d \phi}{d t}\right\rangle\right|_{200}$ for each value of $i_{\mathrm{ac}}$. We call this the dc limit of the Poincaré map and it is pragmatic in explaining our observations. Figure 5(c) shows the dc limit of the map from Fig. 5(b). The chaotic windows average out to noisy regions while the periodic attractors attain their dc values. An attractor to the +1 step is found to occur for $i_{\mathrm{ac}} \in(1.23,1.33)$. Figure 5(c) shows a closeup of the dc Poincaré map of this attractor. As $i_{\mathrm{ac}}$ is increased in this range, we move from being on an attractor with $\left\langle\frac{d \phi}{d t}\right\rangle=0$ to $\left\langle\frac{d \phi}{d t}\right\rangle=+1$ and back to zero again, separated by noisy regions due to the chaotic windows.

The symmetry argument made in the last section implies that the attractor in the range $i_{\mathrm{ac}} \in(1.23,1.33)$ also has solutions corresponding to the -1 step. Which attractor the system settles in depends on the initial conditions. However, the presence of noise removes any bias corresponding to the initial conditions and causes the system to switch between \pm 1 attractors. Figure 5(e) shows this effect, where a noise term with $\sigma_{n}=4 \times 10^{-2}$ is included. It is useful to recall the basin structure, where each set of initial conditions leads to one of the attractors. The presence of noise causes a drift in initial conditions after every period and so it becomes possible for the solution to switch from one attractor to another.

Figures 5(f) and $5(\mathrm{~g})$ show the histogram of experimentally measured $V(t)$ values measured as a function of rf power in the broken node region $(f, B)=6.322 \mathrm{GHz}, B=0$ (f) and $6.350 \mathrm{GHz}, B=0.5 \mathrm{mT}(\mathrm{g})$. We see that the observed 
(a)

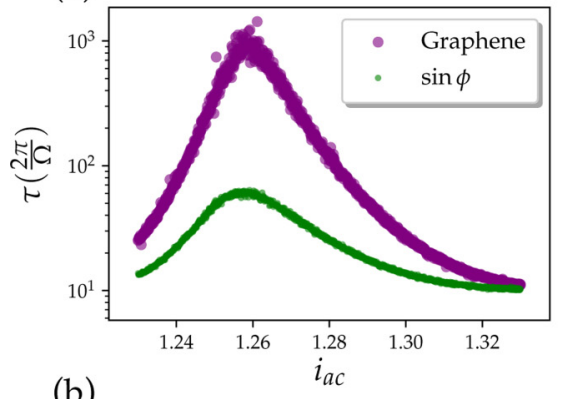

(b)

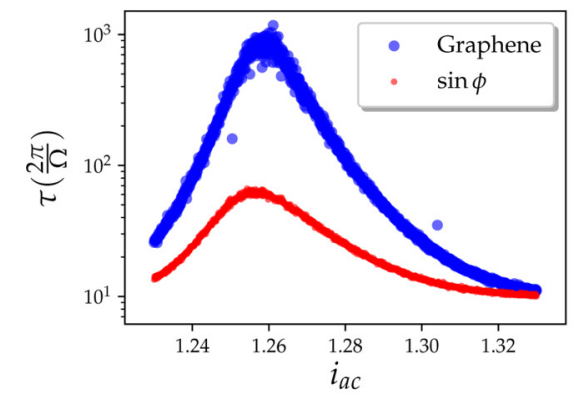

(c)

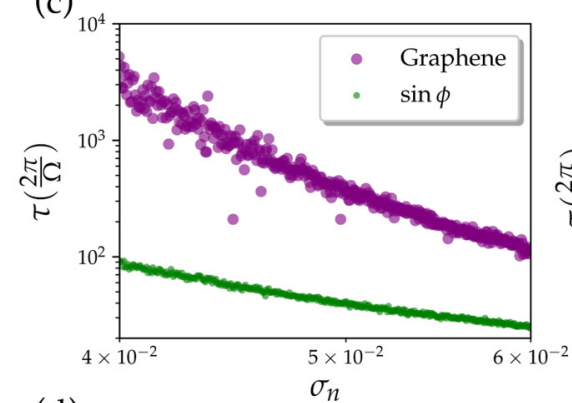

(d)

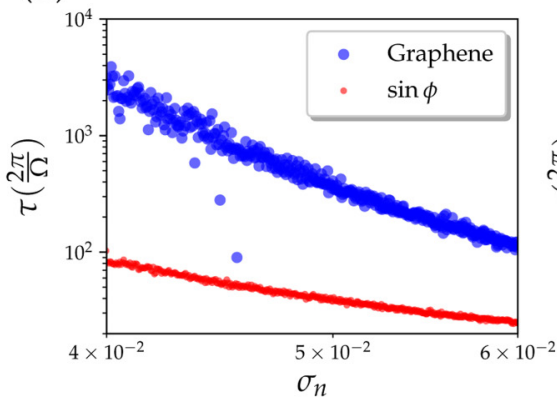

(e)

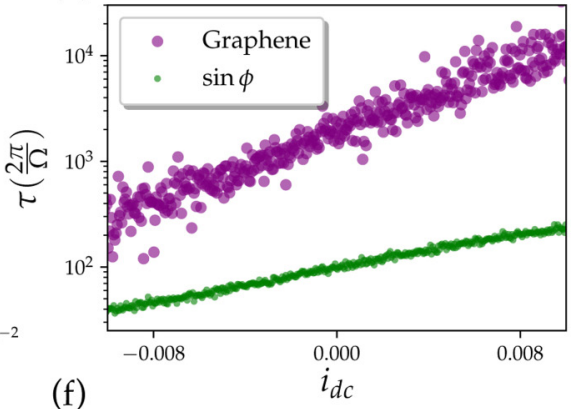

(f)

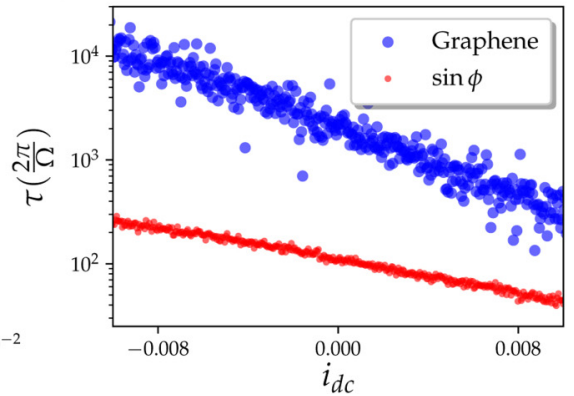

FIG. 6. (a, b) The simulated evolution of switching time on a bistable attractor of the +1 (a) and -1 (b) steps for $i_{\mathrm{dc}}=0$ and $\sigma_{n}=4 \times 10^{-2}$. (c, d) $\tau$ as a function of $\sigma_{n}$ at $i_{\mathrm{dc}}=0$ and $i_{\mathrm{ac}}=1.28$ for the +1 (c) and -1 (d) steps. For low noise levels, $\tau$ becomes very large and decreases with increased noise. (e, f) Effect of $i_{\mathrm{dc}}$ on $\tau$ for the +1 (e) and -1 (f) steps. As $i_{\mathrm{dc}}$ is increased from negative to positive values, the solution prefers to stay more on the +1 step than on the -1 step; the corresponding changes in $\tau$ are evident. In all the plots, the effect of the graphene on sinusoidal CPR is compared. Though both CPRs show a scaling of $\tau$ with $i_{\text {ac }}$, the overall magnitude is larger for the graphene CPR. A window size of ten periods was used to take the dc limit and the system was simulated for a total of $10^{5}$ periods.

behavior of the dc voltage in the broken node region is qualitatively similar to the dynamics in the dc limit of Poincaré maps in Figs. 5(b)-5(e). An attractor to \pm 1 steps is sandwiched between attractors to the zero step separated by noisy regions corresponding to the chaotic windows and leads to the broken node feature observed in the experiment.

\section{F. Scaling of switching time}

Having understood the origin of bistability in simulation, we now focus on the changes in switching time $\tau$ as a function of $\mathrm{dc}$ and ac currents. We reported that $\tau$ undergoes changes over orders of magnitude when $I_{\mathrm{dc}}$ and $\mathrm{rf}$ power are changed in Figs. 3(b) and 3(c). We now reproduce similar scaling in simulation. Figure 6 shows the changes in $\tau$ as a function of $i_{\mathrm{ac}}$, noise level $\sigma_{n}$, and $i_{\mathrm{dc}}$. We use the same set of parameters and the \pm 1 attractor discussed in the last section.

In Figs. 6(a) and 6(b), we plot the switching time $\tau$ for the +1 and -1 steps, respectively. We also compare the effect of the graphene CPR to a simple $\sin (\phi)$ CPR. While fixing all other parameters, the CPR of graphene causes the switching time to be much larger. This feature also occurs with the scaling of switching time as a function of noise level and $i_{\text {dc }}$ [Figs. 6(b)-6(e)]. This can be attributed to the graphene CPR leading to an effective potential for $\phi$ which has steeper and deeper minima, making escape for a given noise level less likely. This increase in $\tau$ resulting from the CPR of graphene serves as an explanation why the bistability might have been harder to directly measure in other underdamped systems.
The presence of chaotic windows for a range of parameters complicates an analytic approach that might elucidate the origin of the observe scaling. Lacking any exact theoretical basis, we now give a heuristic argument for the scaling of the switching time. It is helpful to recall the basin structure picture, where the noise causes a drift of initial conditions, allowing for the solution to switch from the basin of one attractor to another. Formalizing this notion, suppose that the noise causes a diffusion at time $t$ as $\Delta \phi(t) \sim\left(\sigma_{n} \sqrt{t}\right)^{\nu}$, where the term $\sigma_{n} \sqrt{t}$ has been chosen because it is dimensionless and $v$ is a diffusion exponent. $v=1$ corresponds to standard Brownian motion, where net displacement is proportional to $\sqrt{t}$. Suppose $f\left(i_{\mathrm{ac}}, i_{\mathrm{dc}}\right)$ represents a scale in the basin structure; when initial conditions are changed by this scale, a switch from one attractor to another occurs. We can therefore write a condition for the switching timescale $\tau$ :

$$
\Delta \phi(\tau) \sim f\left(i_{\mathrm{ac}}, i_{\mathrm{dc}}\right) .
$$

Using the diffusion expression for $\Delta \phi$, the following expression is obtained:

$$
\tau \sim \frac{f\left(i_{\mathrm{ac}}, i_{\mathrm{dc}}\right)^{1 / v}}{\sigma_{n}^{2}} .
$$

The details of the function $r$ depend on the exact nature of the CPR, noise level $\sigma_{n}$, as well as the parameters $Q$ and $\Omega$. Though we do not have a way to calculate $f\left(i_{\mathrm{ac}}, i_{\mathrm{dc}}\right)$, Eq. (12) show why such relationships might exist in the first place. Further theoretical work is necessary to elucidate the scaling relationship of $\tau$ in the model. 


\section{CONCLUSION AND OUTLOOK}

In summary, we have reported on the ac Josephson in h$\mathrm{BN}$ encapsulated graphene JJs. A number of features distinct from previous measurements from unencapsulated graphene junctions as well as other systems have been observed. The resistive contours separating two steps meet in elongated nodes as opposed to points expected from the RSJ model. Incorporating the capacitance and considering the full RCSJ model in the weakly underdamped limit leads us to provide an origin of these elongated nodes. Moreover, we observe a bistability at the first elongated node. The switching timescale is extraordinarily long, on the order of seconds, and is much slower than any timescale relating to the junction dynamics. A change in $\tau$ over almost three orders of magnitude is observed with changes in external applied dc currents and rf power.

Simulation of the RCSJ model reveals a rich dynamical structure where periodic attractors are interleaved with chaotic windows. In the dc limit, this leads to attractors separated by noisy regions. In particular, we show the presence of an attractor for \pm 1 steps the dynamics of which in the presence of noise is similar to the bistability observed in the broken node region. Simulation of the switching time in the bistable region as a function of $i_{\mathrm{ac}}, i_{\mathrm{dc}}$, and noise level offers an explanation of the scaling behavior observed in the experiment. We also show how the graphene CPR effects the overall magnitude of the switching timescale. We offer a heuristic argument for the existence of such scaling from the interplay between the basin structure and the noise level.

The graphene CPR used in our analysis assumes ballistic transport. It is certainly possible that corrections to this model are necessary and direct measurement of the CPR, for instance, using a superconducting quantum interference device, might be appropriate for the RCSJ model. We have used the ballistic graphene CPR as a prudent choice for this experiment, so as to illustrate why bistability and scaling of the switching timescale are more pronounced in graphene versus other Josephson junctions. However, the conclusions about the nonlinear dynamics hold for a wider class of CPRs including sinusoidal CPRs [45].

A driving theme in contemporary condensed matter research is the realization of Majorana particles and topologically nontrivial ground states [46-49]. One of the principal ways to detect topology in these systems is the $4 \pi$ Josephson effect and it has been used to this effect in certain experiments [7-9]. Yet, chaotic behavior can mimic this effect: the simulation above shows that period doubling is possible for certain values of $i_{\mathrm{ac}}$. Our paper points to a key feature which can indicate the potential for chaotic behavior (i.e., underdamped) in a $\mathrm{JJ}$ - the elongation of nodes in a Shapiro diagram. This seems to be more accurate than the standard means of inferring underdamped junctions, hysteresis, which may be absent in slightly underdamped $(Q \sim 1) \mathrm{JJ}$ s. For example, work on HgTe JJs estimates an overdamped junction from device parameters [50] and as a result rules out the possibility of chaotic behavior in the JJs [8]. The observed elongated nodes contradict this assessment. Further, gaps in resistive nodes have been ascribed to a $4 \pi$-periodic contribution to the CPR $[10,12]$. In this paper, we observe this gap to arise entirely from nonlinear behavior in the junction [Figs. 4(c) and 4(d)].
As the coupling between the superconductor and the material forming the weak link is improved and as the mobility of the weak link is increased, larger values of $I_{C}$ and $Q$ are expected. Hence, a systematic delineation of phase dynamics of driven JJs is crucial for using the ac Josephson effect as a probe of topology and exotic physics.

The underdamped RCSJ model is dissipative with a strength parametrized by $\frac{1}{Q}$. In the limit $Q \gg 1$, the RCSJ model becomes a conservative Hamiltonian system. We have let $\phi$ be a classical variable, though it is possible to quantize the system. It would be interesting to study the quantum dynamics of a Hamiltonian system resulting from the RCSJ model. Further theoretical work in this direction is necessary to understand the full parameter space of the RCSJ model in this limit.

In conclusion, driven JJs where nonlinear effects can lead to the presence of periodic attractors and chaos offer a new route to study nonlinear phenomena. The graphene junction presented in this paper is a tunable platform well suited for such studies. We hope it will be a useful toolbox to study novel phenomena at the intersection of nonlinear dynamics and condensed matter physics.

\section{ACKNOWLEDGMENTS}

We thank Edward Ott and Gleb Finkelstein for useful conversations, and Andrew Seredinski for assistance with sample characterization. S.S.K. gratefully acknowledges financial support from the JQI-QuICS Lanczos graduate fellowship. This work is supported by Army Research Office Grant No. W911NF-18-2-0075 (F.Y. and J.R.W.) and the Physics Frontier Center at the Joint Quantum Institute (Grant No. PHY-1430094) (M.T.W.). Growth of hexagonal boron nitride crystals was supported by the Elemental Strategy Initiative conducted by the MEXT, Japan and the CREST (Grant No. JPMJCR15F3), JST (K.W. and T.T.), and Army Research Office Grant No. W911NF-16-1-0132 (M.H.-R. and F.A).

S.S.K. and F.Y. contributed equally to this work.

\section{APPENDIX A: VARIATION IN THE SHAPIRO DIAGRAM OF THE BROKEN NODE}

In order to understand the origin of the broken node region, we make small changes in the external parameters that affect the junction physics. Figure 7 shows the changes in the broken node region for small changes in $f, V_{\mathrm{BG}}$, and magnetic field. In Fig. 7(a), from left to right, $f$ increases from 6.310, 6.320, 6.330, and $6.370 \mathrm{GHz}$ while $V_{\mathrm{BG}}$ is fixed to $\mathrm{CNP}$ and $B=$ 0. In Fig. 7(b), from left to right, $V_{\mathrm{BG}}$ changes as -0.55 , $-0.85,-1.05$, and $-1.15 \mathrm{~V}$ while $f$ is fixed to $6.350 \mathrm{GHz}$. In Fig. 7(c), we ramp the magnetic field to $0.3 \mathrm{mT}$, fix $f$ to $6.350 \mathrm{GHz}$, and change $V_{\mathrm{BG}}$ as $-0.45,-0.55,-0.65$, and $-0.75 \mathrm{~V}$.

\section{APPENDIX B: SIMULATED SHAPIRO DIAGRAM IN THE OVERDAMPED REGIME}

A junction is said to be overdamped if the capacitance in the RCSJ model is small. In the limit $C=0$, we can rewrite 

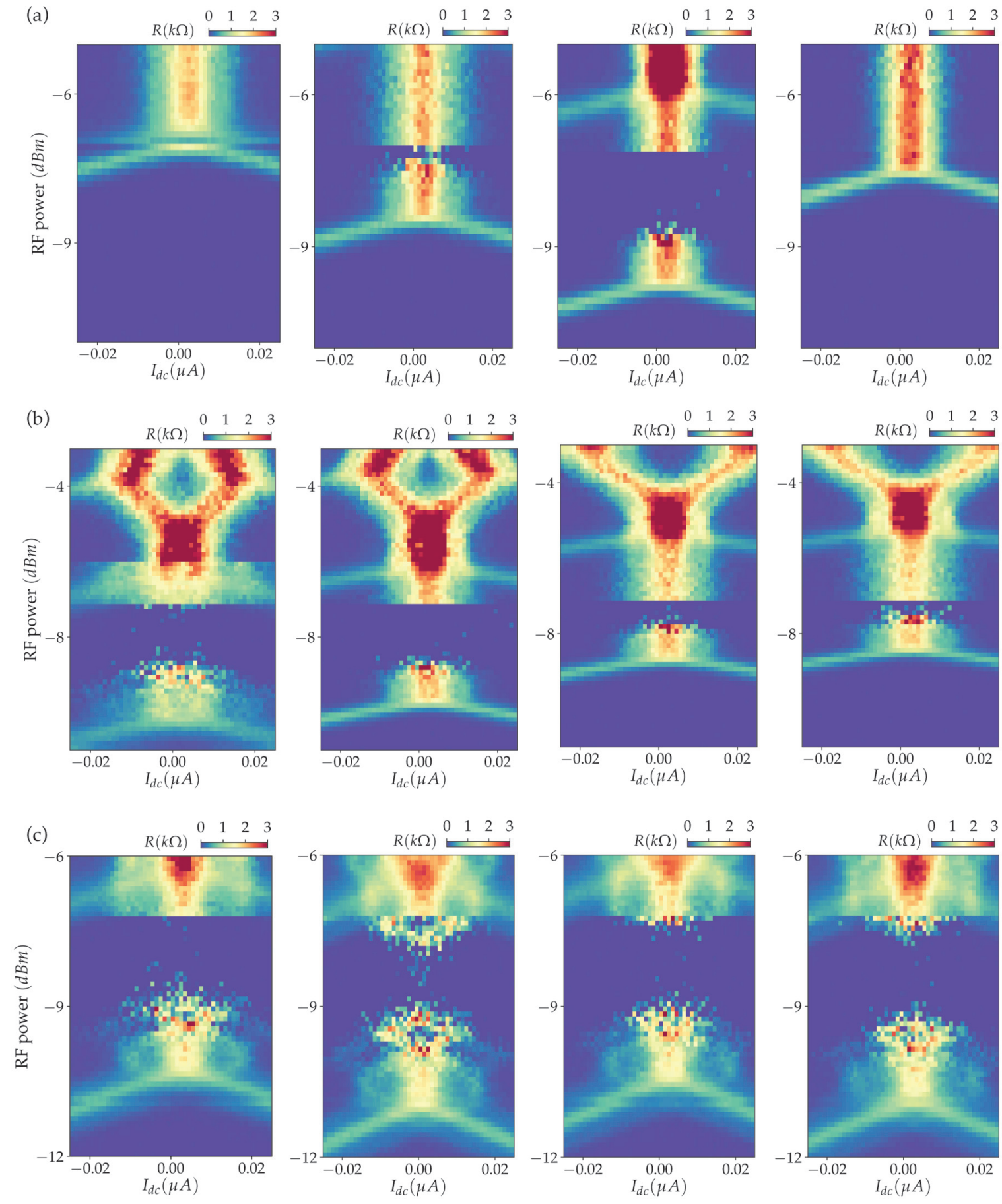

FIG. 7. (a) Left to right: $f$ changes as $6.310,6.320,6.330$, and $6.370 \mathrm{GHz}$ while $V_{\mathrm{BG}}$ is fixed to CNP and no magnetic field is applied. (b) Left to right: $V_{\mathrm{BG}}$ changes as $-0.85,-0.95,-1.05$, and $-1.15 \mathrm{~V}$ while $f$ is fixed to $6.350 \mathrm{GHz}$. (c) $B=0.3 \mathrm{mT}, f$ is fixed to $6.350 \mathrm{GHz}$, and we change $V_{\mathrm{BG}}$ as $-0.45,-0.55,-0.65$, and $-0.75 \mathrm{~V}$.

the evolution equation for $\phi$ as

$$
\frac{\hbar}{2 e R} \frac{d \phi}{d t}+I(\phi)=I_{\mathrm{dc}}+I_{\mathrm{ac}} \sin (\omega t) .
$$

We recast this equation into a dimensionless form by transforming $t \rightarrow t \sqrt{\frac{\hbar}{2 e R I_{c}}}$ :

$$
\frac{d \phi}{d t}+i(\phi)=i_{\mathrm{dc}}+i_{\mathrm{ac}} \sin (\Omega t)
$$

where $\Omega=\omega \sqrt{\frac{\hbar}{2 e R I_{c}}}$ is a normalized frequency. Note that, as compared to the full RCSJ model [Eq. (4)], this system defines a flow in a two-dimensional phase space and its dynamical behavior is constrained to take on fixed points and limit cycles by the Poincaré-Bendixson theorem [24,35]. In fact, it is possible to solve this equation analytically, and the Shapiro step widths take a form given by Bessel functions [1]. For completeness, we reproduce Shapiro diagrams as a function 

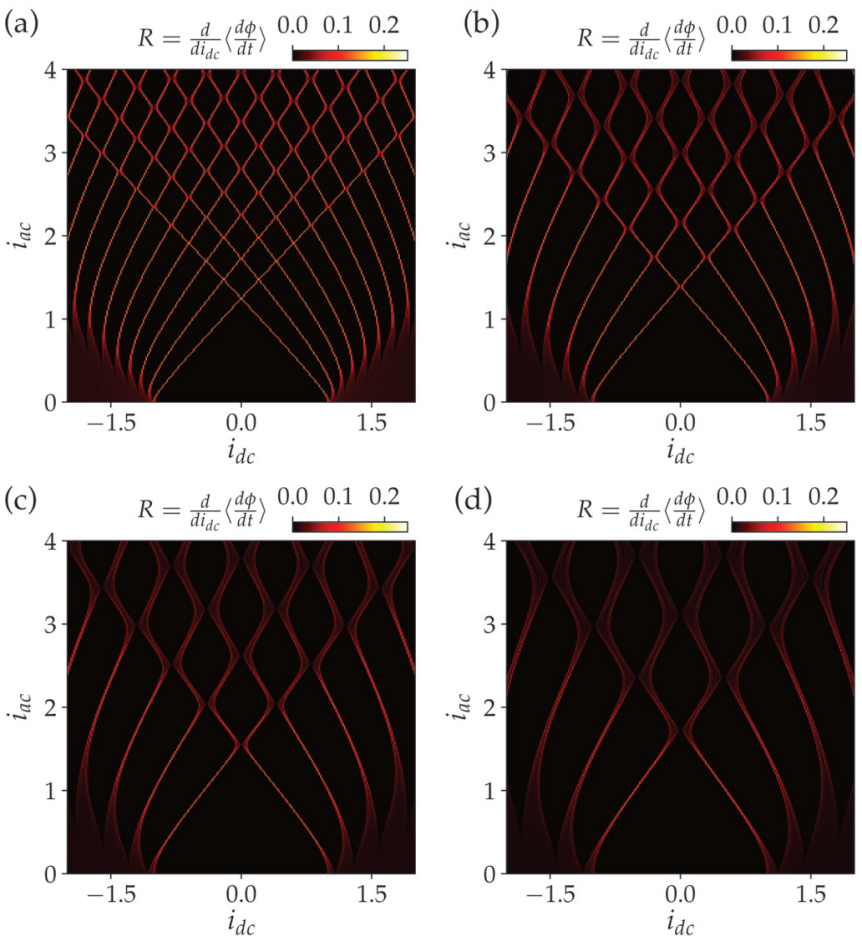

FIG. 8. Simulated Shapiro diagrams in the overdamped limit. From (a) to (d), we plot the differential resistance as a function of $i_{\mathrm{dc}}$ and $i_{\text {ac }}$ for an overdamped model with normalized frequency $\Omega$ taking on values $0.2,0.3,0.4$, and 0.5 , respectively. The resistive contours defining the steps meet in single points.

of varying frequency $\Omega$ in Fig. 8. Such overdamped diagrams have been observed before in graphene junctions [32].

\section{APPENDIX C: UNDERDAMPED SHAPIRO DIAGRAMS}

In addition to the broken node feature described in the main text, the RCSJ model in the weakly underdamped limit $(Q \sim 1)$ can also be used to understand the features in the resistive contours separating the Shapiro steps. Figures 9(a) and 9(c) show a simulated Shapiro diagram with dc voltage and differential resistance respectively. Figure 9(b) shows linecuts at fixed $i_{\mathrm{ac}}$ values. In addition to integer steps, fractional steps are also seen in the transition region between two integer steps. The parameters used for this simulation were $Q=1.5, \Omega=0.5$, and this is a closeup version of the diagram in Fig. 4(a) near the intersection of the \pm 1 step. Figures 9(d) and $9(\mathrm{e})$ show measured Shapiro diagrams at $f$ of $4.000 \mathrm{GHz}$ and $V_{\mathrm{BG}}$ set to CNP and $-0.45 \mathrm{~V}$, respectively. The opening and closing up of the resistive contours in the measurement are qualitatively similar to the fine features observed for $i_{\mathrm{ac}} \in$ $(1.2,1.6)$ in the simulation.

\section{APPENDIX D: ADDITIONAL DATA ON SWITCHING TIME SCALING WITH rf POWER}

The nature of the scaling relationship of switching time $\tau$ with rf power on the bistable step varies with applied frequency and the magnetic field. Figures 10(a) and 10(b) show $\tau$ vs rf power for $\left(f, B, I_{\mathrm{dc}}\right)=(6.338 \mathrm{GHz}, 0 \mathrm{mT}, 0 \mathrm{nA})$ and $\left(f, B, I_{\mathrm{dc}}\right)=(6.35 \mathrm{GHz}, 0.5 \mathrm{mT}, 1 \mathrm{nA})$, respectively. The back gate was set to CNP.
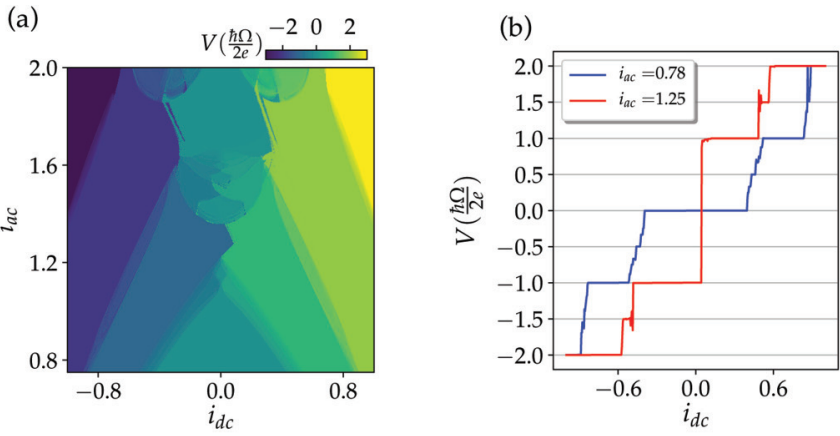

(c)
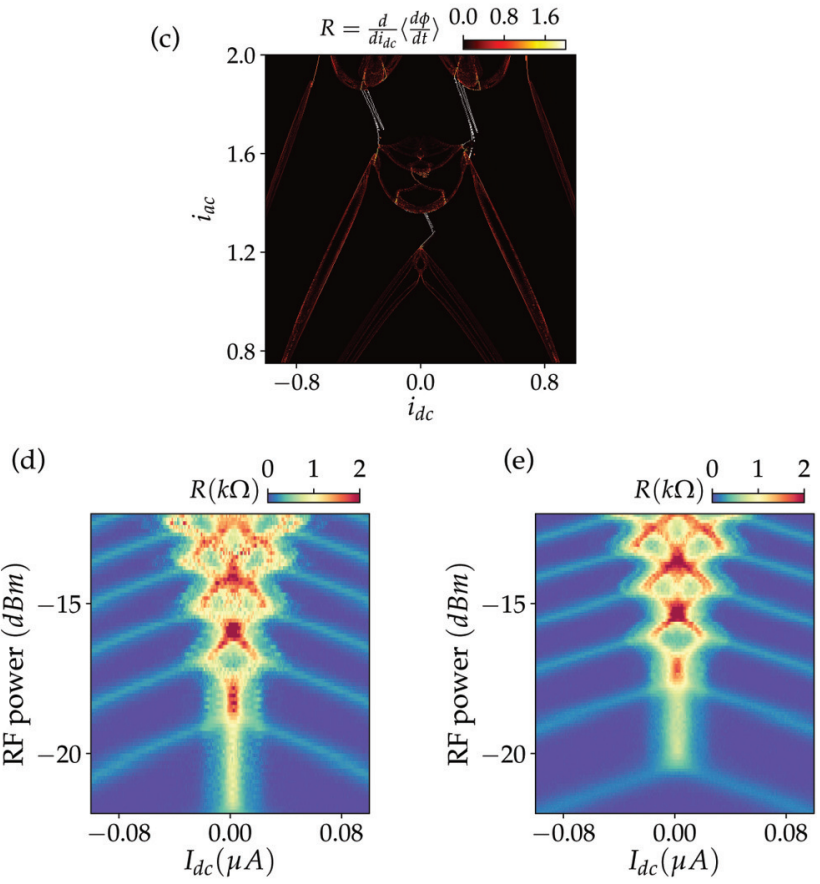

FIG. 9. Underdamped Shapiro diagrams. (a) Simulated dc voltage as a function of $i_{\mathrm{dc}}$ and $i_{\mathrm{ac}}$ in the weakly underdamped limit for the RCSJ model, with $Q=1.5, \Omega=0.5$. (b) Linecuts of the Shapiro diagram in (a) at fixed $i_{\text {ac }}$ values. Both integer and fractional steps are seen. (c) Simulated differential resistance Shapiro diagram for the same parameters as (a). (d, e) Experimentally measured Shapiro diagrams at $4.000 \mathrm{GHz}$, with no magnetic field and $V_{\mathrm{BG}}$ set to CNP and $-0.45 \mathrm{~V}$, respectively.
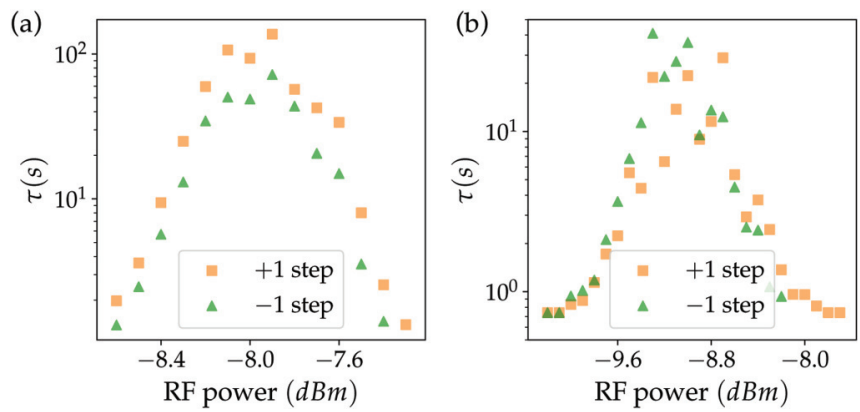

FIG. 10. (a) Switching time $\tau$ vs rf power on the bistable step for $\left(f, B, I_{\mathrm{dc}}\right)=(6.338 \mathrm{GHz}, 0 \mathrm{mT}, 0 \mathrm{nA})$. (a) $\tau$ vs rf power on the bistable step for $\left(f, B, I_{\mathrm{dc}}\right)=(6.35 \mathrm{GHz}, 0.5 \mathrm{mT}, 1 \mathrm{nA})$. 
[1] M. Tinkham, Introduction to Superconductivity (Courier, New York, 2004).

[2] B. D. Josephson, Phys. Lett. 1, 251 (1962).

[3] K. K. Likharev, Dynamics of Josephson Junctions and Circuits (CRC, Boca Raton, FL, 1986).

[4] A. Barone and G. Paterno, Physics and Applications of the Josephson Effect (Wiley, New York, 1982).

[5] M. H. Devoret and R. J. Schoelkopf, Science 339, 1169 (2013).

[6] D. J. Van Harlingen, Rev. Mod. Phys. 67, 515 (1995).

[7] L. P. Rokhinson, X. Liu, and J. K. Furdyna, Nat. Phys. 8, 795 (2012).

[8] J. Wiedenmann, E. Bocquillon, R. S. Deacon, S. Hartinger, O. Herrmann, T. M. Klapwijk, L. Maier, C. Ames, C. Brüne, C. Gould et al., Nat. Commun. 7, 10303 (2016).

[9] D. Laroche, D. Bouman, D. J. van Woerkom, A. Proutski, C. Murthy, D. I. Pikulin, C. Nayak, R. J. J. van Gulik, J. Nygård, P. Krogstrup et al., Nat. Commun. 10, 245 (2019).

[10] A.-Q. Wang, C.-Z. Li, C. Li, Z.-M. Liao, A. Brinkman, and D.P. Yu, Phys. Rev. Lett. 121, 237701 (2018).

[11] W. Yu, W. Pan, D. L. Medlin, M. A. Rodriguez, S. R. Lee, Z.-q. Bao, and F. Zhang, Phys. Rev. Lett. 120, 177704 (2018).

[12] K. Le Calvez, L. Veyrat, F. Gay, P. Plaindoux, C. B. Winkelmann, H. Courtois, and B. Sacépé, Commun. Phys. 2, 4 (2019).

[13] A. A. Golubov, M. Y. Kupriyanov, and E. Il'ichev, Rev. Mod. Phys. 76, 411 (2004).

[14] W. C. Stewart, Appl. Phys. Lett. 12, 277 (1968).

[15] D. E. McCumber, J. Appl. Phys. 39, 3113 (1968).

[16] M. Titov and C. W. J. Beenakker, Phys. Rev. B 74, 041401(R) (2006).

[17] B. A. Huberman, J. P. Crutchfield, and N. H. Packard, Appl. Phys. Lett. 37, 750 (1980).

[18] R. L. Kautz, J. Appl. Phys. 52, 3528 (1981).

[19] R. L. Kautz, J. Appl. Phys. 52, 6241 (1981).

[20] N. F. Pedersen and A. Davidson, Appl. Phys. Lett. 39, 830 (1981).

[21] M. Cirillo and N. F. Pedersen, Phys. Lett. A 90, 150 (1982).

[22] R. L. Kautz, J. Appl. Phys. 58, 424 (1985).

[23] R. L. Kautz and R. Monaco, J. Appl. Phys. 57, 875 (1985).

[24] E. Ott, Chaos in Dynamical Systems (Cambridge University, Cambridge, England, 2002)

[25] J. G. Kroll, W. Uilhoorn, K. L. van der Enden, D. de Jong, K. Watanabe, T. Taniguchi, S. Goswami, M. C. Cassidy, and L. P. Kouwenhoven, Nat. Commun. 9, 4615 (2018).

[26] J. I.-J. Wang, D. Rodan-Legrain, L. Bretheau, D. L. Campbell, B. Kannan, D. Kim, M. Kjaergaard, P. Krantz, G. O. Samach, F. Yan et al., Nat. Nanotechnol. 14, 120 (2019).
[27] F. Amet, C. T. Ke, I. V. Borzenets, Y.-M. Wang, K. Watanabe, T. Taniguchi, R. S. Deacon, M. Yamamoto, Y. Bomze, S. Tarucha et al., Science 352, 966 (2016).

[28] S. Shapiro, A. R. Janus, and S. Holly, Rev. Mod. Phys. 36, 223 (1964).

[29] M. Veldhorst, M. Snelder, M. Hoek, T. Gang, V. K. Guduru, X. L. Wang, U. Zeitler, W. G. v. d. Wiel, A. A. Golubov, H Hilgenkamp et al., Nat. Mater. 11, 417 (2012).

[30] V. S. Pribiag, A. J. A. Beukman, F. Qu, M. C. Cassidy, C. Charpentier, W. Wegscheider, and L. P. Kouwenhoven, Nat. Nanotechnol. 10, 593 (2015).

[31] R. A. Snyder, C. J. Trimble, C. C. Rong, P. A. Folkes, P. J. Taylor, and J. R. Williams, Phys. Rev. Lett. 121, 097701 (2018).

[32] H. B. Heersche, P. Jarillo-Herrero, J. B. Oostinga, L. M. K. Vandersypen, and A. F. Morpurgo, Nature (London) 446, 56 (2007).

[33] C. W. J. Beenakker, Rev. Mod. Phys. 80, 1337 (2008).

[34] J.-D. Pillet, C. H. L. Quay, P. Morfin, C. Bena, A. L. Yeyati, and P. Joyez, Nat. Phys. 6, 965 (2010).

[35] J. Guckenheimer and P. Holmes, Nonlinear Oscillations, Dynamical Systems, and Bifurcations of Vector Fields, Applied Mathematical Sciences (Springer, New York, 2002).

[36] Y. Taur and P. L. Richards, J. Appl. Phys. 48, 1321 (1977).

[37] D. D'Humieres, M. R. Beasley, B. A. Huberman, and A. Libchaber, Phys. Rev. A 26, 3483 (1982).

[38] E. G. Gwinn and R. M. Westervelt, Phys. Rev. Lett. 54, 1613 (1985).

[39] M. T. Levinsen, R. Y. Chiao, M. J. Feldman, and B. A. Tucker, Appl. Phys. Lett. 31, 776 (1977).

[40] E. G. Gwinn and R. M. Westervelt, Phys. Rev. A 33, 4143 (1986).

[41] M. Iansiti, Q. Hu, R. M. Westervelt, and M. Tinkham, Phys. Rev. Lett. 55, 746 (1985).

[42] M. Octavio and C. R. Nasser, Phys. Rev. B 30, 1586 (1984).

[43] Q. Hu, Technical Report No. 25, Division of Applied Sciences, Harvard University, 1987.

[44] A. Davidson, B. Dueholm, and M. R. Beasley, Phys. Rev. B 33, 5127 (1986).

[45] R. L. Kautz, Rep. Prog. Phys. 59, 935 (1996).

[46] F. Wilczek, Nat. Phys. 5, 614 (2009).

[47] F. Wilczek and S. Esposito, in The Physics of Ettore Majorana: Theoretical, Mathematical, and Phenomenological (Cambridge University Press, Cambridge, 2014), pp. 279-302.

[48] R. M. Lutchyn, J. D. Sau, and S. Das Sarma, Phys. Rev. Lett. 105, 077001 (2010).

[49] Y. Oreg, G. Refael, and F. von Oppen, Phys. Rev. Lett. 105, 177002 (2010)

[50] J. B. Oostinga, L. Maier, P. Schüffelgen, D. Knott, C. Ames, C. Brüne, G. Tkachov, H. Buhmann, and L. W. Molenkamp, Phys. Rev. X 3, 021007 (2013). 